\title{
Correlation between structure and resistance of composites based on polystyrene and multilayered graphene oxide
}

\author{
M. N. Nikolaeva ${ }^{1}$, T. D. Anan'eva ${ }^{1}$, A. N. Bugrov ${ }^{1,2}$, A. T. Dideikin ${ }^{3}$, E. M. Ivankova ${ }^{1,3}$ \\ ${ }^{1}$ Institute of macromolecular compounds RAS, Bolshoy pr. 31, 199004 St. Petersburg, Russia \\ ${ }^{2}$ Saint Petersburg Electrotechnical University "LETI", \\ ul. Professora Popova 5, 197376 St. Petersburg, Russia
}

${ }^{3}$ Ioffe Institute RAS, Polytehnicheskaya ul., 26 St. Petersburg, 194021, Russia

marianna_n@mail.ru, anthracene@hq.macro.ru, alexander.n.bugrov@gmail.com, dideikin@mail.ioffe.ru, elena.Ivankova@mail.ioffe.ru

PACS 36.20.-r; 68.65.Pq; 71.20.Rv; 72.80. Tm; 74.78.-w, 68.37.Hk, 73.40.-c

DOI 10.17586/2220-8054-2017-8-2-266-271

\begin{abstract}
The correlation between the structure, resistance and UV-irradiation impact on conductivity of polystyrene-based composites with multilayered graphene oxide flakes was observed. It is established that composites structure and conducting properties depend on concentration, surface modification and the methods by which graphene oxide was incorporated into the polystyrene matrix.
\end{abstract}

Keywords: graphene oxide flakes, polystyrene, composite, conductivity, UV-irradiation.

Received: 3 February 2017

Revised: 24 February 2017

\section{Introduction}

It is known polymer/graphene nanocomposites show superior electrical properties in comparison to unfilled polymers [1-4], so the conductivity level for polystyrene/graphene nanocomposites can reach even more than $20 \mathrm{~S} \cdot \mathrm{m}^{-1}$ [4]. Moreover, recent experimental observations demonstrate that the graphene-based composite synthesized in [5] displays superconducting features at temperatures higher than that of liquid helium and even in the structures $\mathrm{Cu} / \mathrm{Graphene-based} \mathrm{composite/Cu}[6,7]$ without superconducting electrodes. Since graphene is a monolayer of graphite, it is important to compare the electrical properties of graphene with those of graphite. To date, a significant number of indirect experiments indicating the existence of a superconducting state up to room temperature in graphite-based compounds have been reported [8-12]. The main problem of these indirect observations lies in the fact that the superconducting regions occupy only a small areas of carbon materials, and for this reason, the observable effects of superconductivity are negligible in bulk samples [9]. Evidence for superconductivity in graphite lamellae at temperatures above $150 \mathrm{~K}$ has been demonstrated in [13]. Considering graphene, the classical theory of superconductivity does not predict the occurrence of intrinsic superconductivity in such low-dimensional structure without doping. To promote intrinsic superconductivity in graphene, the authors of [14] suggested reconstructing the electronic density of states by inducing elastic deformations. The strain field in graphene can probably be created by introducing it into a polymer host material [6] when chemical bonds are formed between the graphene and polymer. In this case, one can expect graphene flakes with a variety of different deformations. These specific deformations of graphene may be the reason of superconducting state in graphene-based polymer composites.

The aim of this work was to determine why high conductivity and superconductivity arise in polystyrene (PS)-based films with graphene oxide flakes (GF). Polystyrene was used as a polymer matrix because electrization is most expressed in polymers with high resistivity $[15,16]$. Multilayered GF after heat treatment in an aqueous medium [17] becomes semiconductor and can even have a zero bandgap. After special treatment, GF may copolymerize with styrene [5]. 


\section{Experimental}

The methods, described in $[17,18]$ was used for GF production. Natural crystalline graphite was used as a starting material. After series of chemical reactions in liquid medium, the prepared GF was extracted from an aqueous suspension by aerobic drying at a room temperature. As a result, multilayered GF were obtained (Fig. 1). Surface modification of the GF was carried out by treating 3-(trimethoxysilyl)propyl methacrylate (TMPMA) for subsequent reaction with styrene $[5,19]$. Polystyrene and polymer-GF composites ((PS/TMPMA/GF) ${ }^{\text {syn }}$ and $\left.(\mathrm{PS} / \mathrm{GF})^{\text {syn }}\right)$ were synthesized by free radical polymerization in solution [5]. In order to produce the composites, 3 ampoules were used and $19.5 \mathrm{mg}$ of 2,2' -azobis(2-methylpropionitrile) (AIBN) was dissolved in $4 \mathrm{ml}$ mixture of styrene and toluene $(1: 1$ by volume). The prepared solution $(1 \mathrm{ml})$ was dispensed into each ampoule containing $4.8 \mathrm{mg}$ of GF (1 wt.\%), $4.8 \mathrm{mg}(1 \mathrm{wt} . \%)$ and $14 \mathrm{mg}$ (3 wt.\%) of functionalized GF correspondingly. Then ampoules were purged with argon for $10 \mathrm{~min}$ and sealed, after that the ampoules were sonicated for 0.5 hour. Polymerization was carried out for 28 hours at $70^{\circ} \mathrm{C}$. Polystyrene was prepared in an analogous manner. Polymer yield was $70 \%$. Molecular mass average weight of synthesized polystyrene was determined using viscosimetry method and formulae from [19] as 50000. These composites were also obtained by mechanical mixing of PS with GF and PS with functionalized GF: (PS/GF) ${ }^{m i x}$ and (PS/TMPMA/GF) ${ }^{m i x}$.
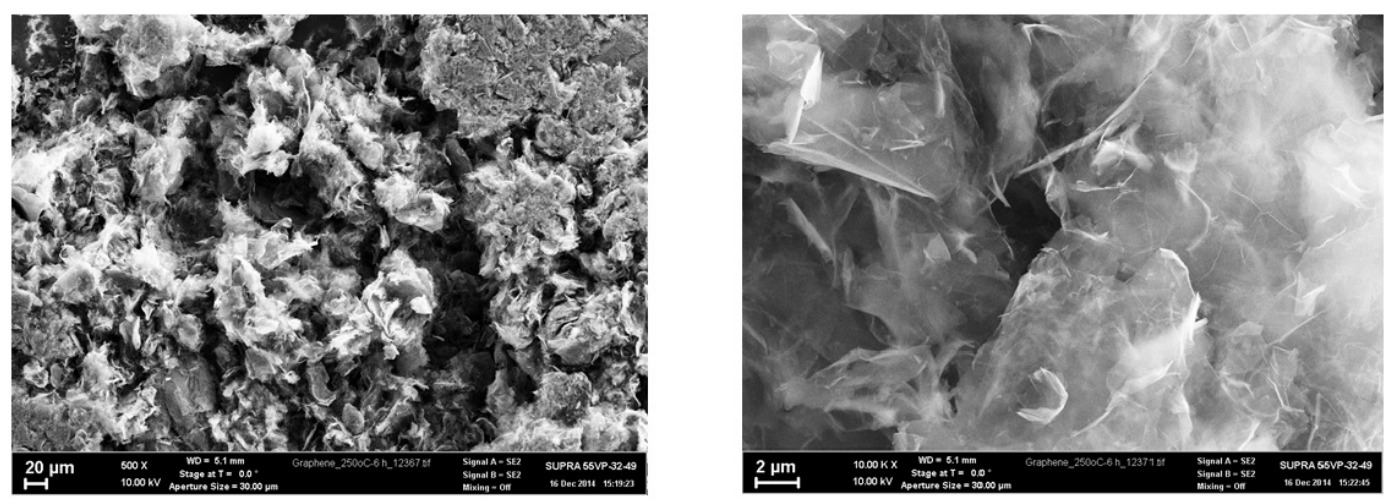

FIG. 1. SEM images of graphene oxide flakes

The distribution and agglomeration of GF particles in a polystyrene matrix was investigated by Zeiss Supra 55VP field emission scanning electron microscope (Germany). Cross sections of polystyrene-GF films for SEM investigation were prepared by cryofracture in liquid nitrogen.

Films of the polymer composite were deposited on copper electrodes through the dispenser by casting from the 1 wt.\% solution. Thickness of the films was controlled by the interference microscope using the method described in [20-22]. Current-voltage characteristics of the metal-composite-metal structures were obtained by the modified two-probe method. The area of electrodes was $1 \mathrm{~cm}^{2}$. Measurements were carried out at the pressure not higher than $1 \mathrm{~kg} / \mathrm{cm}^{2}$. The resistive nature of obtained current-voltage characteristics exclude the presence of a breakdown in films which have been investigated.

UV- irradiation of the composite films was performed by full spectrum of Hg-lamp DKB-9 with energy $220 \mathrm{~kW}$ and having effective wavelength range of $185-270 \mathrm{~nm}$.

\section{Results and discussion}

The surfaces of polystyrene films are rather smooth, even for very thin films. The surface of polystyrene films without graphene oxide is presented in Fig. 2a,b. The conductivity of polystyrene films for was observed only on thickness not higher than $0.02 \mu \mathrm{m}$. Surface topographies of (PS/GF) mix film cleavages are presented in Fig. 2c,d. For (PS/GF) ${ }^{\text {syn }}$ and (PS/TMPMA/GF) ${ }^{m i x}$, SEM images are similar. As revealed by SEM (Fig. 2f,g) in the case of the (PS/TMPMA/GF) ${ }^{s y n}$ composite with $1 \mathrm{wt} . \%$ of GF, the inclusions of GF are distributed rather evenly in the polystyrene matrix obviously due to the formation of chemical bonds between the graphene and polystyrene. For the mechanically-mixed (PS/TMPMA/GF) ${ }^{m i x}$ and composite with $3 \mathrm{wt} \%$ of functionalized GF (PS/TMPMA/GF-3) ${ }^{\text {syn }}$, the GF particles are not distributed in significant amount on the surface of the composite film (Fig. 2c,d,h,i). Therefore, the conductivity values for these films do not increase significantly compared to pure polystyrene, as it can seen from current-voltage characteristics (Fig. 3). Apparently, GF particles in $(\mathrm{PS} / \mathrm{TMPMA} / \mathrm{GF})^{m i x}$, (PS/GF) ${ }^{m i x}$ and $(\mathrm{PS} / \mathrm{GF})^{\text {syn }}$ composites have a large chemical affinity for the polymer 


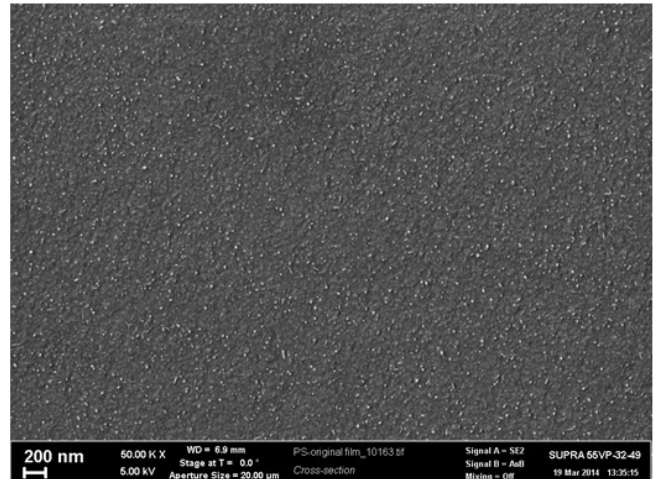

a

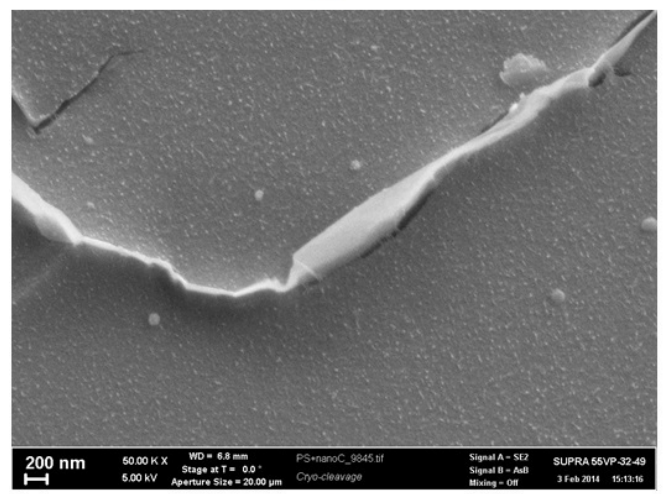

c

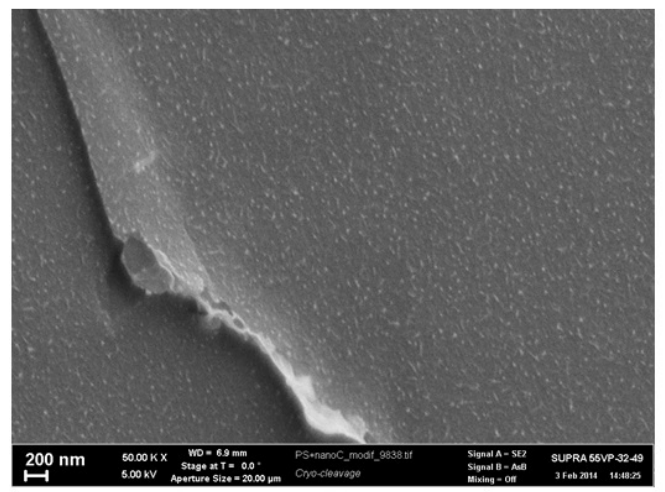

f

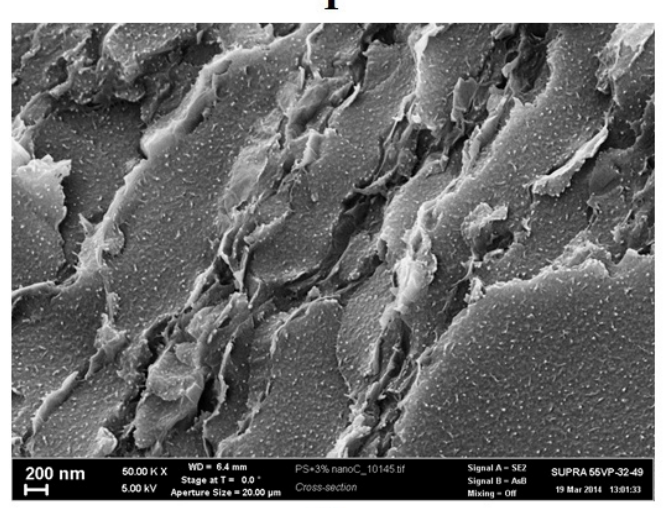

h

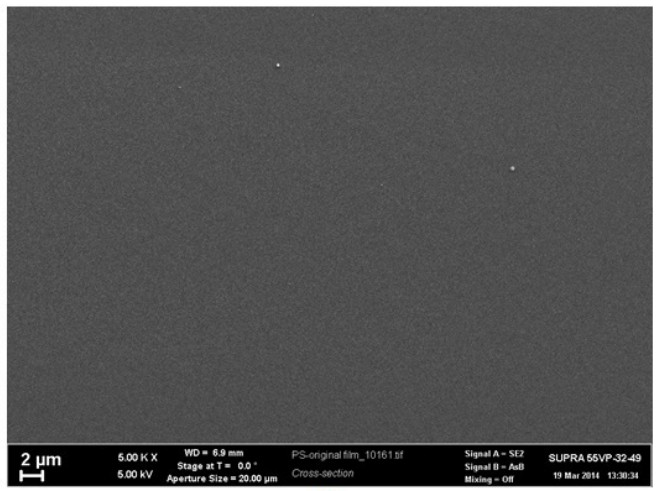

b
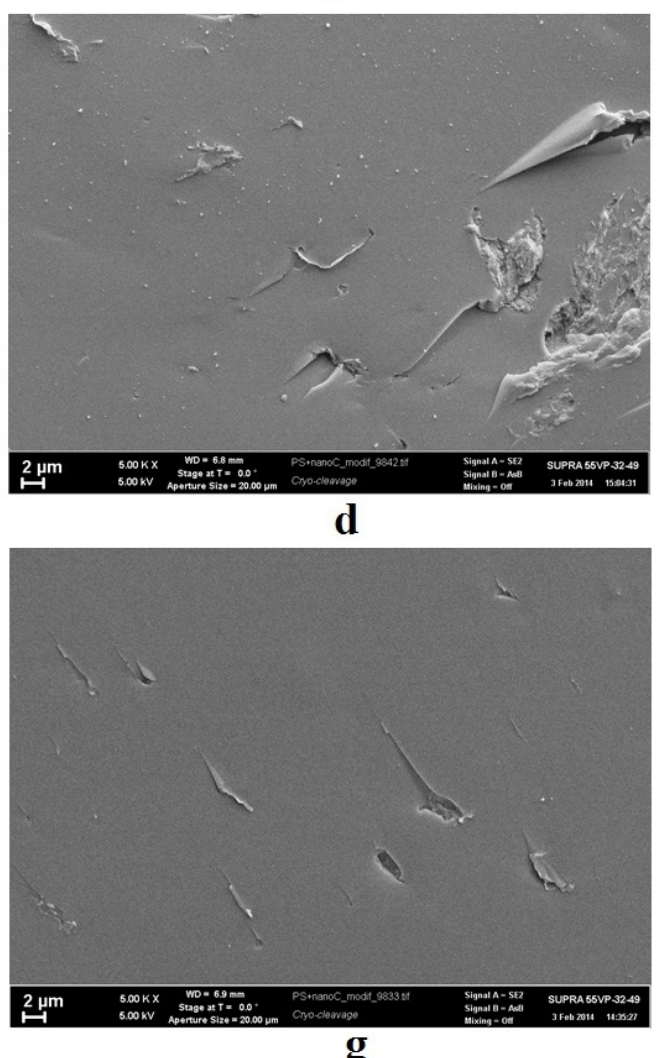

g

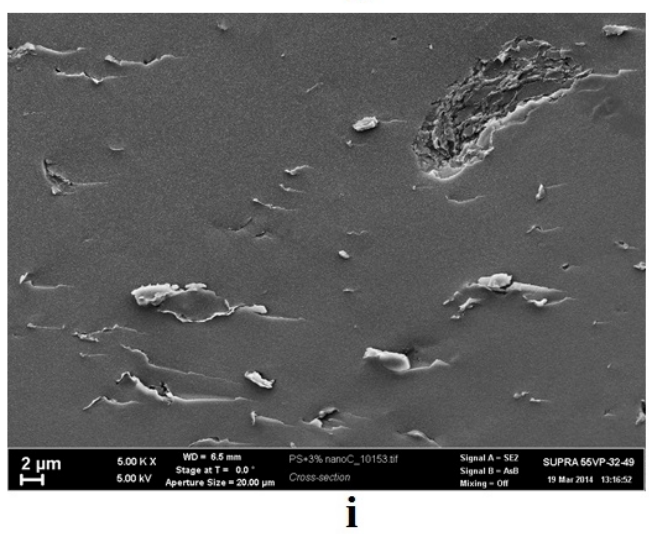

FIG. 2. SEM images of the polystyrene (a, b), (PS/GF) ${ }^{m i x}(\mathrm{c}, \mathrm{d}),(\mathrm{PS} / \mathrm{TMPMA} / \mathrm{GF})^{\text {syn }}(\mathrm{f}, \mathrm{g})$, $(\mathrm{PS} / \mathrm{TMPMA} / \mathrm{GF}-3)^{\text {syn }}(\mathrm{h}, \mathrm{i})$ films 


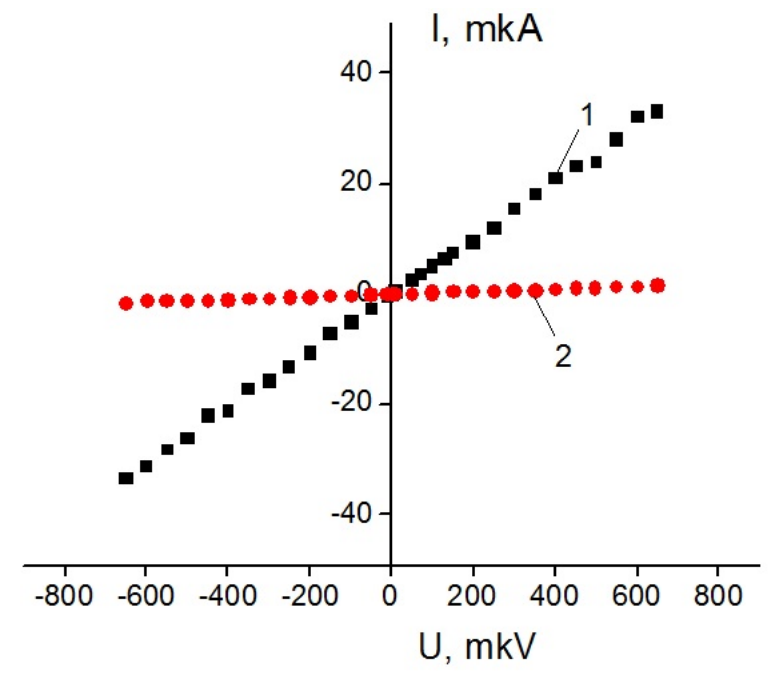

FIG. 3. Typical current-voltage characteristics for $\mathrm{Cu} / \mathrm{GF}$ composite/Cu systems, 1 - for $(\mathrm{PS} / \mathrm{TMPMA} / \mathrm{GF})^{\text {syn }}$ and $2-\mathrm{f}$ or $(\mathrm{PS} / \mathrm{GF})^{\text {syn }}$, films thickness $2 \mu \mathrm{m}$

matrix of polystyrene and are preferentially immersed in it completely, simultaneously leaving defects on the polymer surface. Films of (PS/GF) ${ }^{\text {mix }}$ demonstrate random GF inclusions on the surface which are fully covered by polystyrene (Fig. 2c,d). The surface topography of (PS/TMPMA/GF-3) syn at the same time drastically changes from that of others composites' surfaces probably due to the excess of GF which did not form covalent bonds with polystyrene matrix completely and directed to agglomeration under van der Waals forces. For the case of composites (PS/GF) ${ }^{\text {syn,mix }}$ as also for (PS/TMPMA/GF) ${ }^{\text {mix }}$, conductivity was low as it can be seen from current-voltage characteristics (Fig. 3, curve 2). It is worth noting that the GF concentration rise in (PS/TMPMA/GF) $^{\text {syn }}$ (from 1 to $3 \mathrm{wt} . \%$ ) significantly increased films resistance up to values of tens of $\mathrm{k} \Omega$ as it was for (PS/TMPMA/GF) ${ }^{m i x}$, (PS/GF) ${ }^{m i x}$ and (PS/GF) ${ }^{\text {syn }}$ composites. To the contrary as it was shown earlier [19], composite films (PS/TMPMA/GF) syn with thickness less than $3 \mu \mathrm{m}$ conduct electric current and for $(\mathrm{PS} / \mathrm{TMPMA} / \mathrm{GF})^{\text {syn }}$ ohmic behavior of current-voltage characteristic is observed indicating a metallic type of conductivity.

Observations of the composites' SEM images and their resistance values show that in fact the systems under considerations could be divided into 3 categories: 1) polystyrene, 2) (PS/TMPMA/GF) ${ }^{\text {syn }}$ and 3) $(\mathrm{PS} / \mathrm{TMPMA} / \mathrm{GF})^{m i x},(\mathrm{PS} / \mathrm{GF})^{m i x},(\mathrm{PS} / \mathrm{GF})^{\text {syn }},(\mathrm{PS} / \mathrm{TMPMA} / \mathrm{GF}-3)^{\text {syn }}$. So we can suppose resistance of composite films is dependent on composites structure and consequently on surface topography. The preparation methods of composites obviously also have an influence on the formation of films' surface. Composite (PS/TMPMA/GF) $^{\text {syn }}$ with low resistance demonstrates specific distribution of GF on films surface that substantiate the formation of chemical bonds between styrene and GF during synthesis. Obviously in this case, the chemical bonds formed between GF and PS prevent mutual adhesion of graphene sheets and create substantial stretches and deformations for erasing high conductivity. The scheme of GF modification and evident chemical reaction with styrene is shown in Fig. 4.

After that, measurements of resistance of GF composites films were performed before and after UV-irradiation. UV-irradiation was carried out for 2 hours for films of $2 \mu \mathrm{m}$ thickness. The resistance was lowered by 1 order of magnitude for composites (PS/TMPMA/GF) $)^{\text {syn }}$, being reduced from 20 to $2 \Omega$. This result correlates with measurements of resistance for UV-irradiated graphite in [23]. For (PS/GF) ${ }^{\text {syn, mix }}$, (PS/TMPMA/GF) $^{\text {mix }}$ as for individual GF, the influence of UV-irradiation on films' resistance has not been observed. Resistance value remained unchanged and was near $30 \mathrm{k} \Omega$ for $(\mathrm{GF} / \mathrm{PS})^{m i x, s y n}$ and (PS/TMPMA/ GF) ${ }^{m i x}$ composites. This effect can be explained as the impact of UV-irradiation on GF directly when some edges of GF are situated on films' surface as in the case of (PS/TMPMA/GF) ${ }^{\text {syn }}$.

These above-presented results for (PS/TMPMA/GF) ${ }^{\text {syn }}$ can be explained by the formation of covalent bonds firmly holding GF particles in given positions; then some edges of the GF are distributed on the surface of polystyrene film. Additionally, during the synthesis and formation of covalent bonds between the polystyrene and GF special mechanical stresses can be created. As known, they contribute to the emergence of superconductivity [6, 7], predicted for graphite [8-12]. 


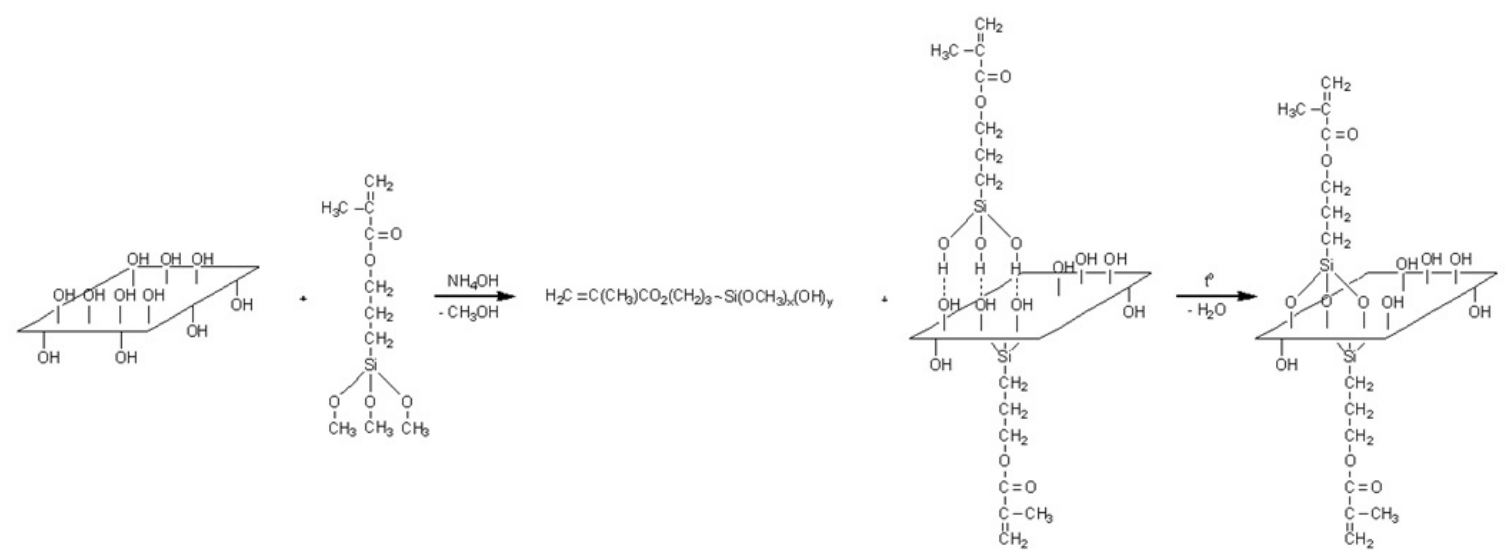

a

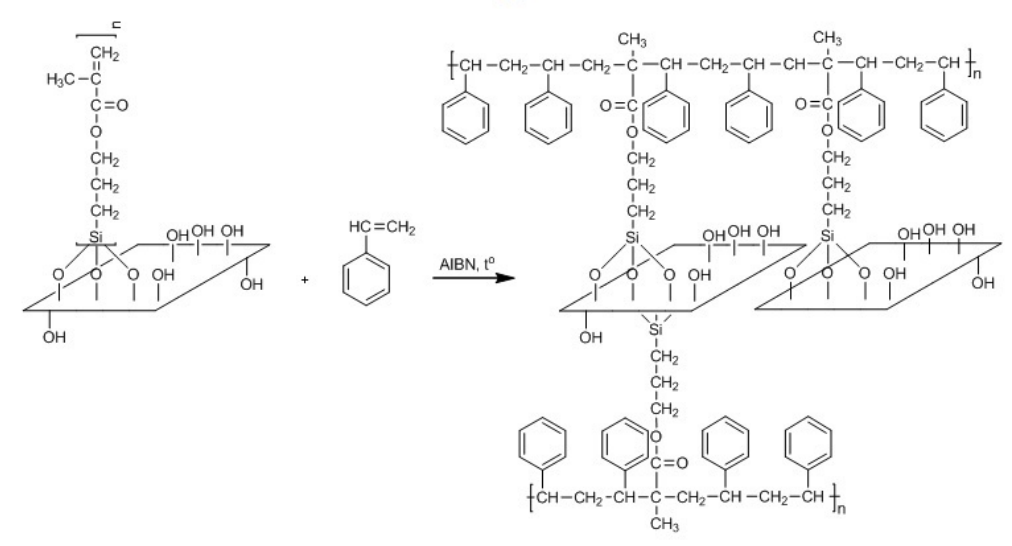

b

FIG. 4. Procedure of GF surface modification (a) and subsequent synthesis with styrene (b)

\section{Conclusions}

The differences in the conductivity of composites based on polystyrene with chemically bonded and unbonded GF have been determined. The films of (PS/TMPMA/GF) ${ }^{\text {syn }}$ composite with chemically bonded GF showed metallic type conductivity and demonstrated low resistance up to $2 \mu \mathrm{m}$ thickness. All other composite films had rather high resistance because of lack of conducting GF particles on the surface. Thus, a correlation between the structure of the films and conductive properties was observed. The influence of UV-radiation exposure on the composite films was also found for (PS/TMPMA/GF) $)^{s y n}$, where the direct absorption of radiation by graphene flakes is possible.

\section{References}

[1] Stankovich S., Dikin D.A., et. al. Graphene-based composite materials. Nature, 2006, 442(7100), P. $282-288$.

[2] Eda G., Chhowalla M. Graphene-based composite thin films for electronics. Nano Lett., 2009, 9(2), P. 814-822.

[3] Abramov D., Arakelian S., et al. Interaction of femtosecond laser radiation with carbon materials: exfoliation of graphene structures and synthesis of low-dimensional carbon structures. Nanosystems: Physics, Chemistry, Mathematics, 2016, 7(1), P. $220-225$.

[4] Deepak A., Shankar P. Exploring the properties of lead oxide and tungsten oxide based graphene mixed nanocomposite films. Nanosystems: Physics, Chemistry, Mathematics, 2016, 7(3), P. 502-505.

[5] Nikolaeva M.N., Bugrov A.N., et al. Conductive properties of the composite films of graphene oxide based on polystyrene in a metalpolymer-metal structure. Russ. J. Appl. Chem., 2014, 87(8), P. 1151-1155.

[6] Ionov A.N. Josephson current-voltage characteristic of a composite based on polystyrene and graphene oxide. Tech. Phys. Lett., 2015, 41(7), P. 651-653.

[7] Ionov A.N. Josephson-like behaviour of the current-voltage characteristics of multi-graphene flakes embedded in polystyrene. J. Low Temp. Phys., 2016, 182(3/4), P. 107-114.

[8] Antonowitcz K. Possible superconductivity at room temperature. Nature, 1974, 247(5440), P. 358-360.

[9] Esquinazi P., Garcia N., et. al. Indications for intrinsic superconductivity in highly oriented pyrolytic graphite. Phys. Rev. B., 2008, 78(13), P. $134516(1-8)$. 
[10] Scheike T., Böhlmannet W., et. al. Can doping graphite trigger room temperature superconductivity? Evidence for granular hightemperature superconductivity in water-treated graphite powder. Advanced Materials, 2012, 24(43), P. 5826-5831.

[11] Lebedev S.G. Evidence of josephson-like behaviour of thin granular carbon films. International Review of Physics, 2008, 2(5), P. 312-328.

[12] Felner I., Kopelevich Y. Magnetization measurement of a possible high-temperature superconducting state in amorphous carbon doped with sulfur. Phys. Rev. B, 2009, 79(23), P. 233409(1-4).

[13] Ballestar A., Barzola-Quiquia J., et. al. Josephson-coupled superconducting regions embedded at the interfaces of highly oriented pyrolytic graphite. New J. Phys., 2013, 15(5), P. 023024.

[14] Uchoa B., Barlas Y. Superconducting states in pseudo-Landau levels of strained graphene. Phys. Rev. Lett., 2013, 111(4), P. 046604(1-5).

[15] Lowell J., Rose-Innes A.C. Contact electrification. Adv. Phys., 1980, 29(6), P. 947-1023.

[16] Duke C.B., Fabish T.J. Charge-induced relaxation in polymers. Phys. Rev. Lett., 1976, 37(16), P. 1075-1078.

[17] Mikoushkin V.M., Shnitov V.V., Nikonov S.Yu., et al. Controlling graphite oxide bandgap width by reduction in hydrogen. Tech. Phys. Lett., 2011, 37(10), P. 942-945.

[18] Aleksenskii A.E., Brunkov P.N., Dideikin A.T., et al. Single-layer graphene oxide films on a silicon surface. Tech. Phys., 2013, 58(11), P. 1614-1618

[19] Khairullin A.R., Nikolaeva M.N., Bugrov A.N. Resistance of the composite films based on polystyrene and graphene oxide. Nanosystems: Physics, Chemistry, Mathematics, 2016, 7(6), P. 1055-1058.

[20] Nikolaeva M., Boiko Y., Martynenkov A. Supramolecular structure and conductive properties of dielectric polymers in metal/polymer/metal systems. Int. J. Polym. Mat., 2013, 62(13), P. 706-710.

[21] Nikolaeva M.N., Anan'eva T.D., et al. Influence of chemical structure and chain length on conducting properties of dielectric polymers in metal/polymer/metal structures. Rus. J. Appl. Chem., 2013, 86(5), P. 756-759.

[22] Nikolaeva M.N., Martynenkov A.A., et al. Resistance of dielectric polymer films with fillers in metal-polymer-metal systems. Rus. $J$. Appl. Chem., 2014, 87(5), P. 646-650.

[23] Smirnov V.A., Arbuzov A.A., Shul'ga Yu.M., et al. Photoreduction of graphite oxide. Chemistry of high energies, 2011, 45(1), P. 60-64. 